\title{
Novel Ideal Nonimaging Designs by Multichanneling the Phase-Space Flow
}

\author{
Juan C. Miñano ${ }^{* a b}$, Pablo Benítez ${ }^{\text {ab }}$, Liu Jiayao ${ }^{a}$, José Infante ${ }^{\mathrm{a}}$, Julio Chaves ${ }^{\mathrm{b}}$, Asunción Santamaría ${ }^{\mathrm{a}}$ \\ ${ }^{a}$ Universidad Politécnica de Madrid (Cedint), Campus Montengancedo UPM, 28223 Madrid, Spain; \\ ${ }^{\mathrm{b}}$ LPI, 2400 Lincoln Ave., Altadena, CA 91001, USA
}

\begin{abstract}
New ultra-thin optical designs are presented. They are formed by optical sections (called channels) working in parallel (multichanneling) to provide the desired optical function. The phase-space representation of the bundle of rays going from the source to the target is discontinuous between channels. This phase-space ray-bundle flow is divided in as many branches as channels there are but it is a single trunk at the source and at the target. Typically, these multichannel devices are at least formed by three optical surfaces: two of them have discontinuities (in the shape or in the shape derivative) while the last one is smooth. The discontinuities of the optical surfaces are causing the separation of the flow in branches (in the phase space). The number of discontinuities is the same in the two first surfaces: Each channel is defined by the smooth surfaces in between discontinuities, so the surfaces forming each separate channel are all smooth. No diffractive analysis is done.
\end{abstract}

Keywords: SMS method, thin optics, optical design, lens design, aplanatic

\section{INTRODUCTION}

LEDs are more suitable for compact optics than incandescent lamps or fluorescent lamps. In the first case is because the operating temperature of LEDs allows low cost optics working near the LED while in the second case, the higher radiance of the LEDs makes them more suitable for collimating applications. The interest for thin nonimaging optics components has also showed up in the Concentrating Photovoltaics (CPV) sector [1], [2].

The approach to designing very thin concentrator/collimators has not always been the same. All approaches share the idea of dividing the light flow in different "channels" each one of them having its own optics whose surfaces are not shared with neighbouring channels. At the turn of the century, multichannel devices called Stepped flow-line optics (SFL) were developed [3],- [5]. They are designed with the flow-line method [6] and rapidly found applications in the design of backlights [7], [8], optics for combining light sources or efficiently distributing light to several locations [9] [13]. The main disadvantage of these devices lies in their manufacturability which is in general complex because the flow-line mirrors, derived from the method, use to have complex shapes and large areas (relative to the aperture area).

This paper presents a design procedure for thin optics based in the Simultaneous Multiple Surfaces (SMS) design method [14]-[18], solving the aforementioned disadvantage of flow-line based designs. Nevertheless, flow-lines are sometimes used when they don't create manufacturability complexities. We will describe this procedure by applying it to the design of an LED collimator.

\section{DESIGN OF THE MULTICHANNEL RXI}

The design procedure explained here is restricted to rotational symmetric cases, i.e., they are 2D designs in the sense that only the degrees of freedom of a 2 dimensional design are allowed. The multichannel thin optics based on the SMS method are characterized by having an optical surface called light distributor $d$ which collects the light from a source (an LED in our example) and distributes it over a given support surface. The distributor is in general a mirror. The optics will be confined between two support surfaces whose cross sections are the lines $\ell$ and $m$ which, in this example, are straight lines (see Fig. 1). Fig. 1 also shows the flow-lines of the input and output beams. The input beam is formed by

*jc.minano@upm.es ; phone +34 915441060; fax +34 915446341

International Optical Design Conference 2010, edited by Julie Bentley, Anurag Gupta,

Richard N. Youngworth, Proc. of SPIE-OSA Vol. 7652, 765220 - @ 2010 SPIE

CCC code: $0277-786 \mathrm{X} / 10 / \$ 18 \cdot$ doi: $10.1117 / 12.870596$ 
the rays issuing from the LED and the output beam is the collimated beam that we want to have at the output. In the case of Fig. 1 the flow-lines of the output beam are vertical parallel lines. Once the multichannel RXI is designed, the flow lines must be continuous so every flow line of the input beam will be connected with its corresponding flow line at the output beam. The distributor $d$ is designed so the flow-lines of the input beam, once reflected by the distributor, intercept the "mirror supporting line" $m$ at the point where the flow-lines of the output beam would intercept $m$ if there was a refraction on the surface $\ell$ (see Fig. 2). In this case, since the surface $\ell$ is flat, all we have to do is a straight extension of the the flow lines at the output. In this way, the irradiance (flow per unit of surface) on both supporting surfaces will be the same. This requirement is established to avoid discontinuities on the line 1, which is sometimes necessary to simplify manufacturing, to minimize losses and to avoid corners that can get dirty.

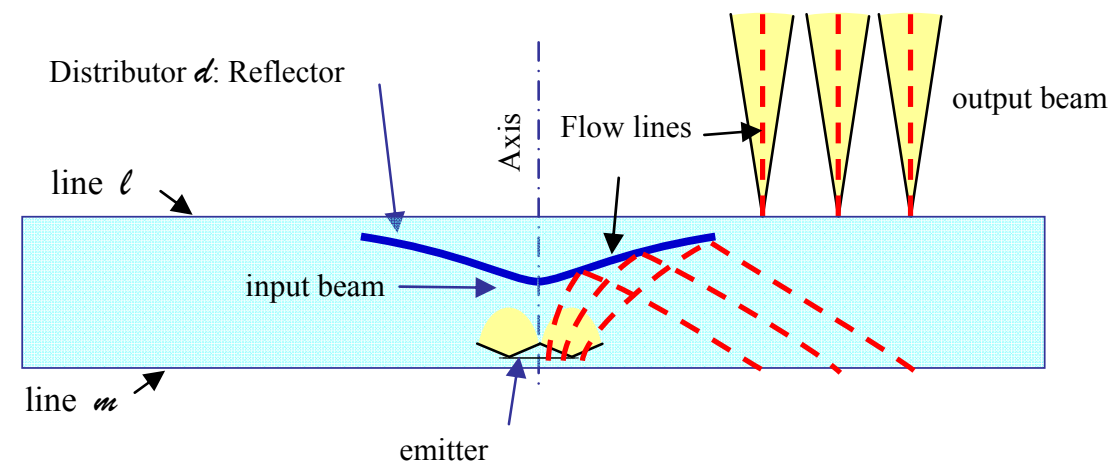

Fig. 1. Design of the distributor $d$. The optical system to design is bounded by lines $\ell$ and $m$.

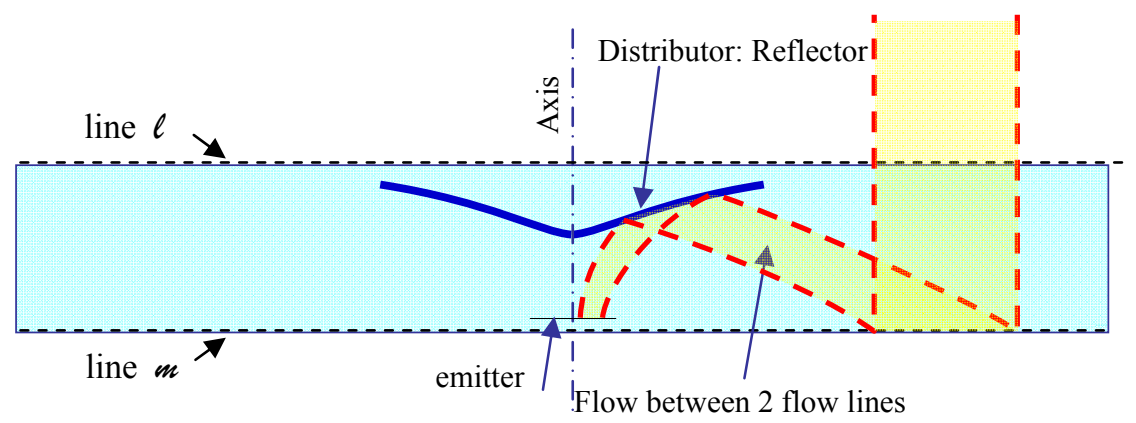

Fig. 2. The distributor ensures that the average irradiance on the supporting surface $m$ is equal to that on the supporting surface $\ell$.

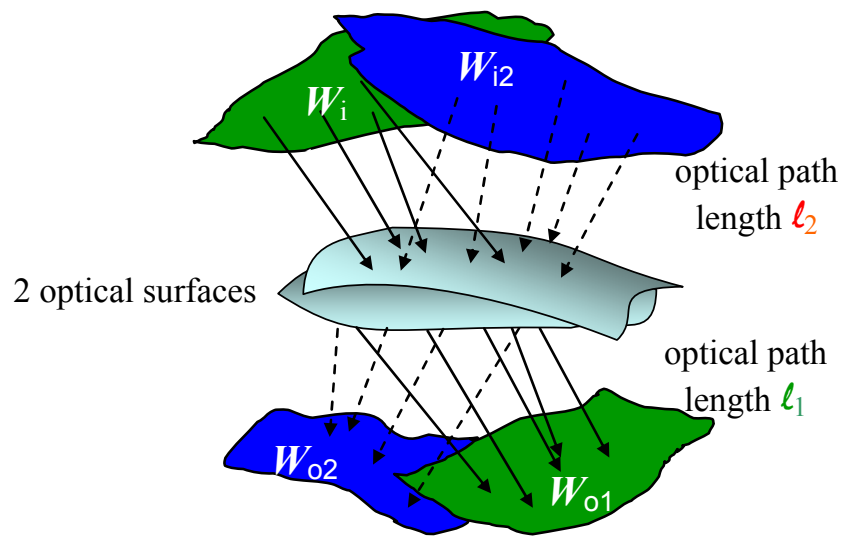

Fig. 3. (a) Cartesian oval transforms one input congruence onto an output congruence. (b) the simplest version of the SMS $3 \mathrm{D}$ method provides two surfaces that transform two input congruences onto two output ones. 
Once the distributor is known, a refractive and a reflective surface transforming the edge rays of the incoming beam onto edge rays of the output beam has to be designed. Since there are two edge rays bundles at the input beam (and 2 at the output beam), this is a classical 2-surfaces SMS design problem. This is schematically shown in Fig. 3, where each edge ray bundle is represented by a wavefront normal to it. As shown in this Figure, the optical path lengths between wavefronts at the input and at the output can be selected. Each selection of optical path lengths gives a different solution of the problem. In general the solutions are mathematically correct but physically possible only in some cases, as it happens in the classical Cartesian oval problem [19], [20] By selecting different optical path lengths we will be able to design the different channels with the condition that the surface be continuous on $\ell$. Fig. 4 shows the final design with the trajectories of some rays. These trajectories show how the bundle is chopped by the mirrors of the multichannel and rebuilt by the lenses.

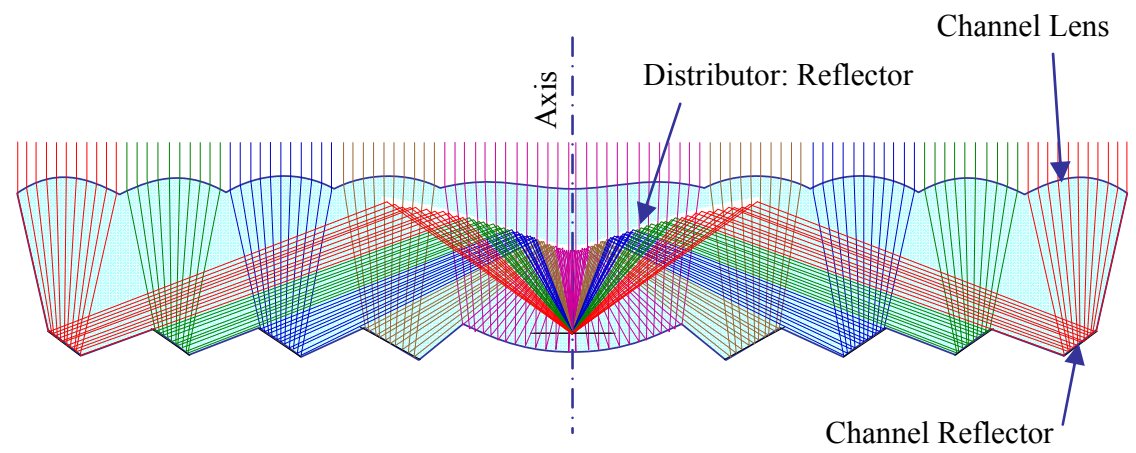

Fig. 4. Multichannel RXI.

\subsection{Aplanatic design}

Aplanatic systems are axisymmetric optical designs free from on-axis spherical aberration and linear coma. These two conditions entail two further design conditions: (1) stigmatic on-axis image and (2) the Abbe sine condition (note that all the rays involved in these conditions are exclusively tangential). The contrary statement is also true: a design forming a stigmatic on-axis image and fulfilling the Abbe sine condition is aplanatic. Aplanatic designs have long been known through the work of Schwarzschild [23], Wassermann and Wolf [24], Welford [25], Mertz [26], [27] and others. Recently Lynden-Bell and Willstrop derived an analytic expression of more general aplanats [28], [29]. The Abbe sine condition can be derived from the condition of zero linear coma, but this is just one possible point of view. For instance, Clausius, who derived the Abbe condition in 1864, did it from thermodynamic arguments [25] (we can also say that he used the conservation of etendue theorem [6]). In 1884, Hockin gave a proof using path differences along tangential rays (without considering sagittal rays) [25].

Aplanatic designs are particular cases of two-surface SMS 2D designs [6], [14] - [19]. An SMS design is a more general case which in its simpler version is capable of forming stigmatic image of two off-axis points. When these two points of the (two-surface) SMS design are at an infinitesimal distance apart around the axis, the SMS design becomes an aplanatic design [16].

Two rotationally symmetric surfaces give enough degrees of freedom to accomplish the Abbe sine condition and the Fermat principle and thus two optical surfaces are enough to get an aplanatic design. The most well known examples are aplanatic lenses (two refractive surfaces) and the Schwarzschild's mirrors. Once the refractive indices of the different media are known, there are only two constants to determine uniquely the two profiles of the aplanatic design. Of course, the optical system may have more than two surfaces. In general it is possible to design an aplanatic system by designing only two of them, i.e., considering all other surfaces prescribed. These other surfaces can be used for different purposes. In this work we use an additional surface (the distributor) to get an ultra thin aplanatic systems with a continuous surface $\iota$ in which the incident wavefronts are broken, focussed and rebuilt in different optical channels that operate in parallel.

The design procedure of an aplanatic multichannel RXI is just a particularization of the procedure described before: The flow-lines become now the on-axis rays, the condition of continuity of the flow lines becomes the Abbe sine condition, the emitter is the focus point $\mathbf{F}$, and the output beam is the beam of parallel on-axis rays. This design procedure is schematically shown in Fig. 5 


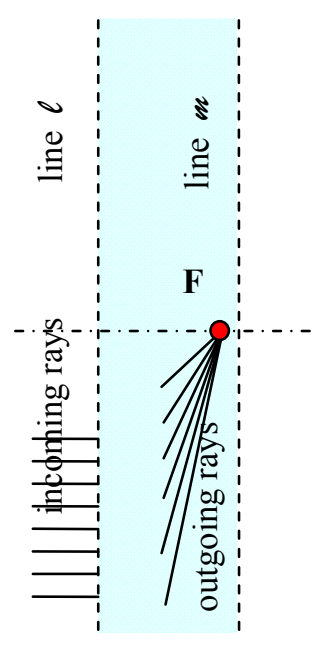

(a)

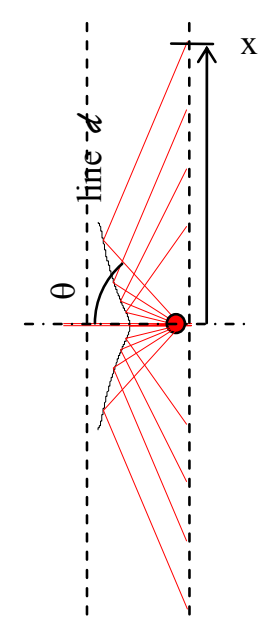

(b)

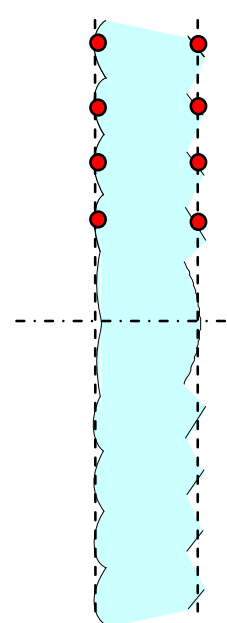

(c)

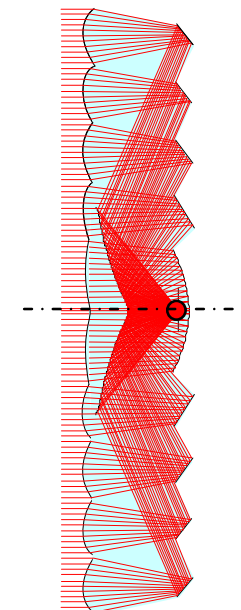

(d)

Fig. 5. Design process of an aplanatic multichannel RXI: (a) point $\mathrm{F}$ and lines $\ell$ and $m$ are choosen, (b) the distributor is designed so the rays from $\mathbf{F}$ after reflection on it find line $m$ at a point with coordinate $x$ such that $x=f \sin \theta$, (c) aplanatic designs of focal lens $f$ are calculated with different optical path lengths so their refractive surfaces are continous and tangent to the line $\ell$. (d) View of the on-axis ray trajectories.

The distributor plays no role in the bundle "cut-and-paste" procedure, i.e., the bundle of an aplanatic lens can be chopped and rebuild without any distributor. This is because the Abbe sine condition can be fulfilled with different optical path lengths, a different one for each channel. This is shown in Fig. 6. The figure shows two aplanatic lenses of the same fnumber. The one on the left has continuous surfaces (one channel) while the one on the right has two channels. Every on axis ray fulfills the Abbe sine condition with the same focal length.

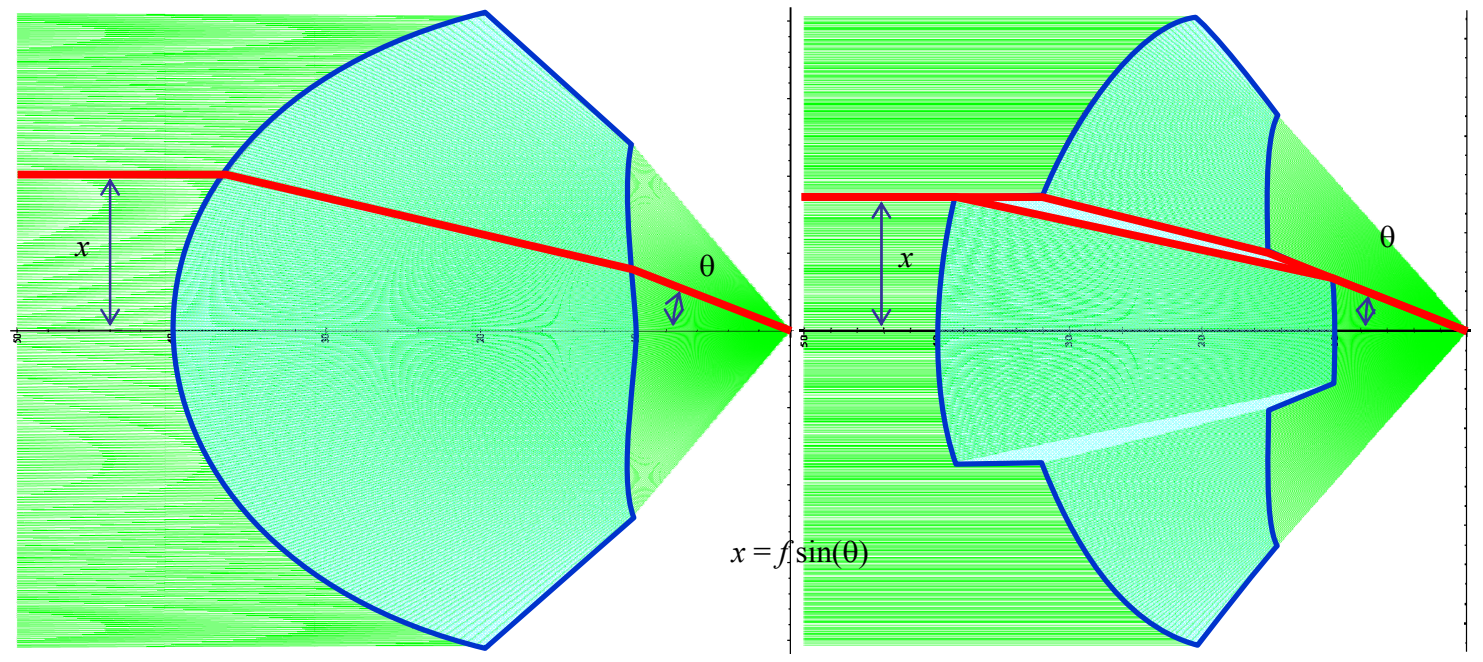

Fig. 6. Continuous aplanatic lens (left) and 2-channel aplanatic lens (right) with the same f-number.

\subsection{Distributor options}

The distributor is often placed so it blocks the trajectory of incoming rays after refraction at the lenses. To avoid this problem there are two solutions that can be used together or alternatively:

1. Eliminate the channels whose incoming rays are blocked by the distributor $d$. This would eliminate the central channel of Fig. 7 and partially its adjacent channel. When the central channels are deleted, the central part of the distributor becomes useless. This is important because this central part could not work by TIR. In most of 
these cases, the usable part of the distributor can work by TIR. Additionally, we can place a lens in the deleted central part of the distributor to help the efficiency. This central part won't have the same degree of collimation as the remaining parts of the multichannel RXI, i.e., in the aplanatic limit, this central part won't have the same focal length.

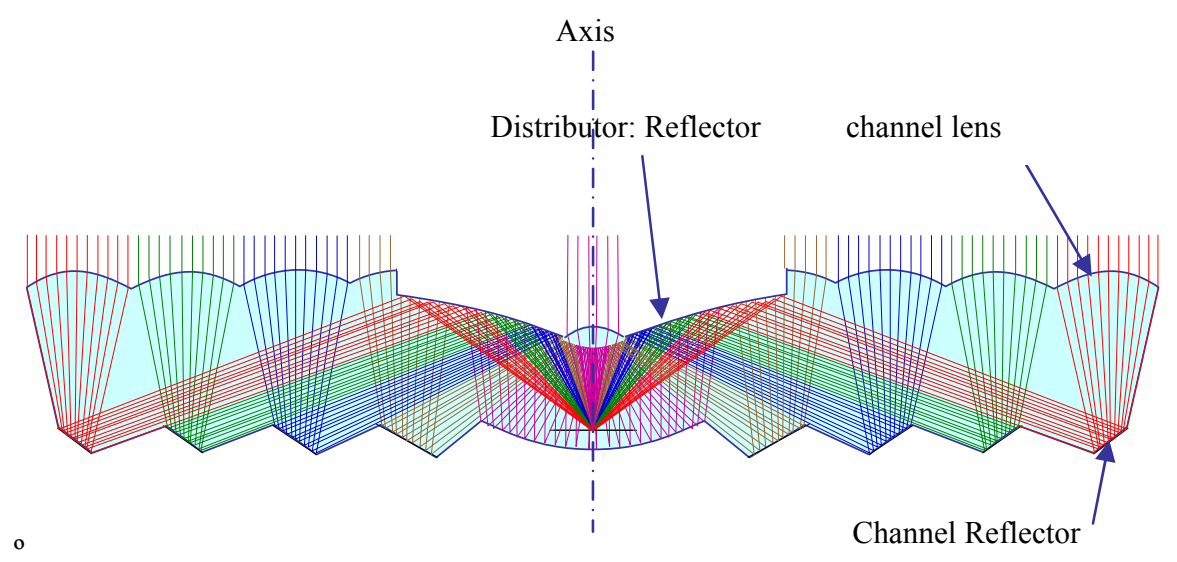

Fig. 7. Option 1: Channels blocked by the distributor are deleted.

2. Create a thin layer of low refractive index at the position of the distributor so it works as a TIR mirror for most of the rays issuing from $\mathbf{F}$ but is transparent for the incoming rays (in a similar way as the front reflector of the SMS 2D design called XX works [6]). This is the case shown in Fig. 4. Again, there is a central part that won't work by TIR. This part can be metalized, which will produce some losses by light blocking (in general very small, $<1 \%)$.

\section{RAY TRACING RESULTS}

Fig. 8 and Fig. 9 show two multichannel RXIs whose inner channels have been deleted according to option 1 of the previous section. They are designed for a Platinum Dragon Osram LED (LWW5SN). There is an air gap between the RXI multichannel and the LED encapsulation so the rays enter the multichannel RXI within $\pm \arcsin (1 / n), n$ being the refractive index $(n \approx 1.49)$. The channel reflectors are assumed to be metalized.

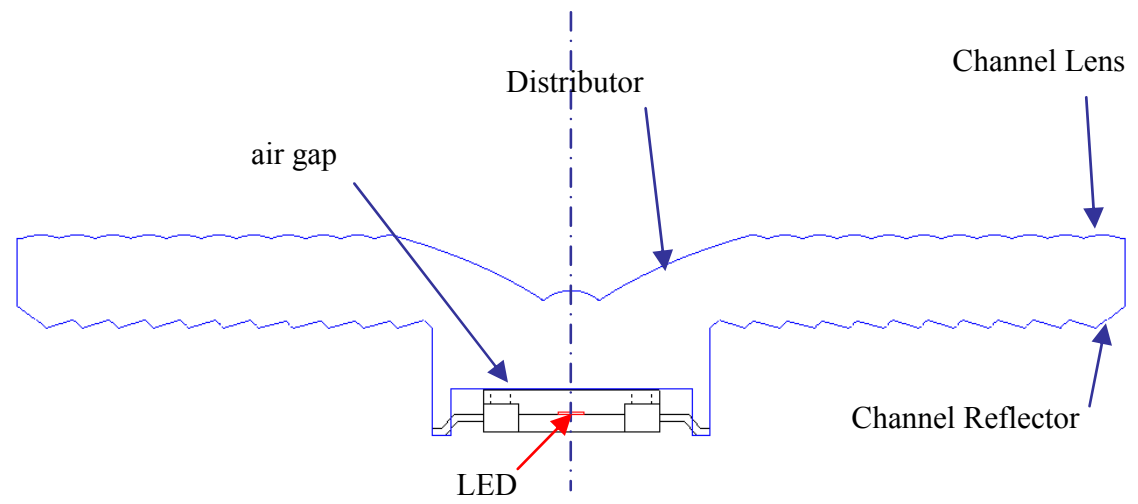

Fig. 8. Multichannel RXI designed for an LED. Emission half angle \pm 2.6 deg. 


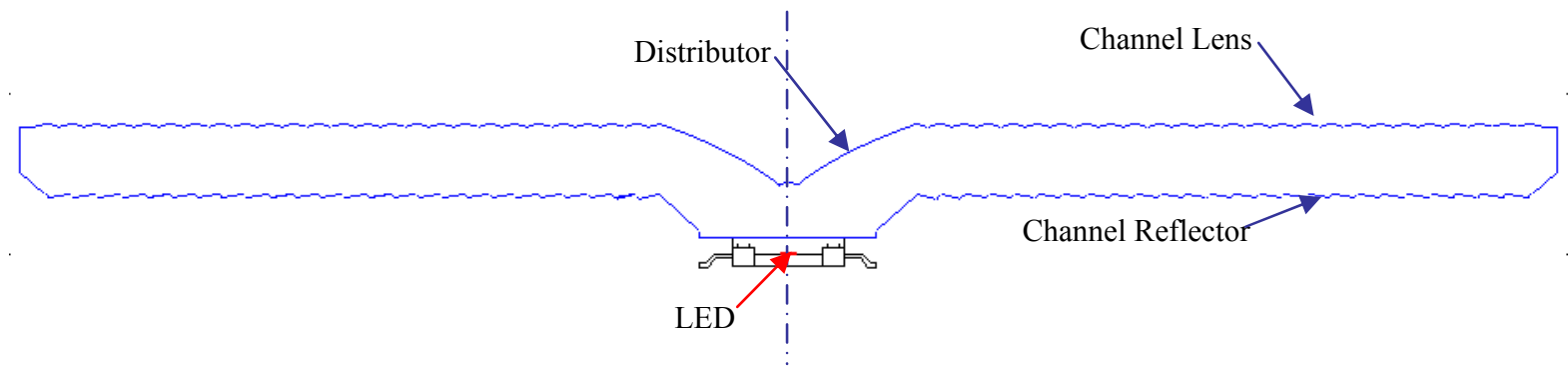

Fig. 9. Multichannel RXI designed for an LED. Emission half angle $\pm 1.5 \mathrm{deg}$.

Preliminary results show that losses due to non collimated light (i.e., losses due to absorption in PMMA, emission through the edges and bottom) is $12 \%$ for the case of Fig. 8 and 18\% for Fig. 9. Fig. 10 shows the intensity pattern of the multichannel RXI of Fig. 9.

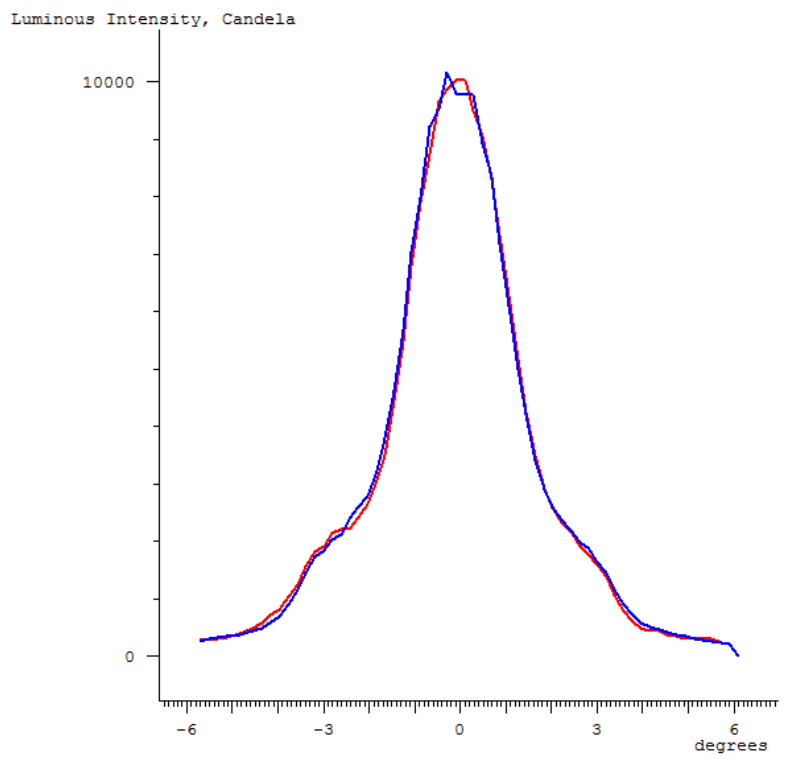

Fig. 10. Intensity pattern of the device of Fig. 9.

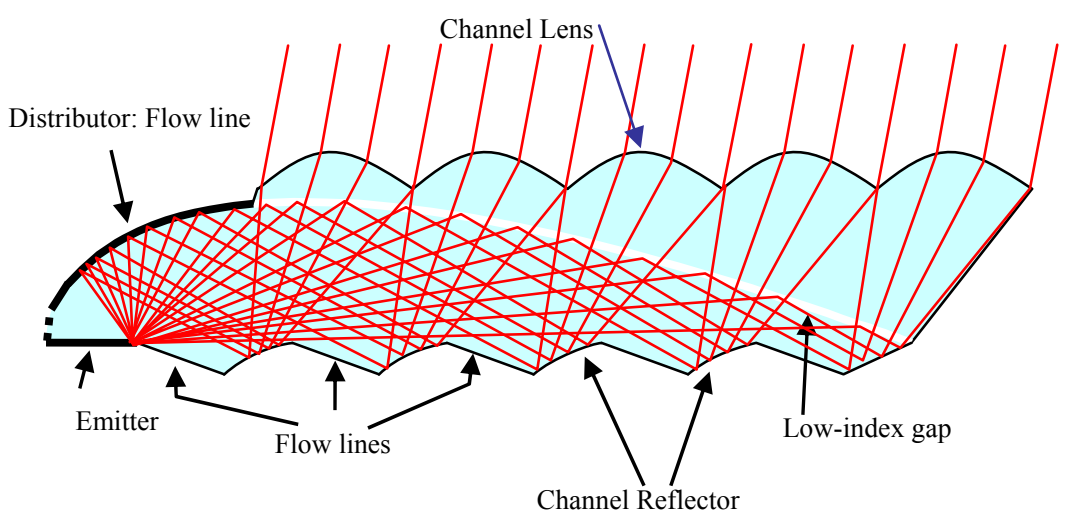

Fig. 11. Example of multichannel RXI whose distributor is a non-sequential mirror designed as a flow-line. This type of distributors require the lines between channel mirrors to be also flow lines for a better collimating performance. 


\section{MULTICHANNEL RXI COMBINED WITH NON SEQUENTIAL MIRRORS}

Thinner devices can be obtained if the distributor is a flow line. In this case, the surface between reflector channels must also be flow lines of the flow emitted by the source. This is shown in Fig. 11. The distributor is designed in the same way as before, i.e., to get continuous flow lines with those obtained by refracting the flow lines at the output on the lens supporting surface. The difference is that this distributor design is now based on flow-lines. Channel lenses and channel reflectors are designed with the SMS method. This multichannel RXI still shares with the preceeding ones its simplicity for manufacturing if we compare them with thin devices whose channels are also designed with the flow-line method.

\section{CONCLUSIONS}

We have presented a procedure to design thin optical devices and in particular thin RXI. The designs contain a distributor element which is used to get the thin optical element and to ensure continuity of one of the surfaces. The remaining optical elements are grouped in channels. In the multichannel RXI each channel comprises a reflector and a lens. The bundle of rays is chopped so each part is processed by a different channel and rebuild at the exit of the channels.

Initial results show that this design concept is an efficient way to collimate light in very thin devices whose manufacturability is improved with respect other thin design alternatives.

\section{ACKNOWLEDGEMENT}

The LPI's devices presented in this paper are protected under US and International patents pending by LPI LLC, 2400 Lincoln Avenue, Altadena, CA 91001 USA http://www.lpi-llc.com/. The authors thank Spanish Ministries MCINN (Sigmasoles PSS-440000-2009-30; Deffio TEC2008-03773, Engineering Metamat. CSD2008-00066), MITYC (Osv: TSI-02303-2008-52), the Madrid Regional Government (Abl: PIE/466/2009, F3: PIE/469/2009) and UPM (Q090935C019) for their partial support. The authors also thank Bill Parkyn for his help in editing the paper

\section{REFERENCES}

[1] Jason H. Karp, Eric J. Tremblay, and Joseph E. Ford, "Planar micro-optic solar concentrator," Opt. Express 18, 1122-1133 (2010) http://www.opticsinfobase.org/oe/abstract.cfm?URI=oe-18-2-1122

[2] http://www.morgansolar.com/fulldescription.php

[3] J. Chaves, M. Collares-Pereira, "Ultra flat ideal concentrators of high concentration", Solar Energy, 69, p.269, (2000)

[4] J. Chaves, M. Collares-Pereira, "Ideal concentrators with gaps", Appl. Opt., 41, p.1267, (2002)

[5] Julio Chaves, Introduction to Nonimaging Optics, CRC Press, 2008

[6] R. Winston, J.C. Miñano, P. Benítez, Nonimaging Optics, (Academic Press, New York, 2005).

[7] J.C. Miñano, P. Benitez, J. Chaves, W. Falicoff, W. Parkyn, "Etende-conserving illuminantion-optics for backlights and frontlights", US patent application

[8] J.C. Miñano, P. Benitez, J. Chaves, M. Hernandez, O. Dross, and A. Santamaria, "High-efficiency LED backlight optics designed with the flow-line method", Nonimaging Optics and Efficient Illumination Systems II, Proc. SPIE, Vol. 5942, 2005

[9] J. Chaves, W. Falicoff, J.C. Minano, P. Benitez, W. Parkyn, R. Alvarez, O. Dross, “Optical manifold for lightemitting diodes", United States Patent 7380962

[10] J. Chaves, W. Falicoff, O. Dross, J.C. Miñano, P. Benitez, W. Parkyn, "Combination of light sources and light distribution using manifold optics", Nonimaging Optics and Efficient Illumination Systems III, SPIE Vol. 6338, 2006

[11] A. Cvetkovic, O. Dross, J. Chaves, P. Benitez, J.C. Miñano, and R. Mohedano, "Etendue-preserving mixing and projection optics for high-luminance LEDs, applied to automotive headlamps", Optics Express, 14, 26, pp. 13014-13020, (2006)

[12] O. Dross, J.C. Miñano, P. Benitez, A. Cvetkovic, J. Chaves, "Non-imaging optics combine LEDs into one bright source", SPIE Newsroom, http://newsroom.spie.org/x3596.xml (2006) 
[13] O Dross, A Cvetkovic, J Chaves, P Benitez, J.C. Minano, "LED Headlight Architecture that creates a High Quality Beam Pattern independent of LED Shortcomings", Nonimaging Optics and Efficient Illumination Systems II, SPIE Vol.5942, p.126-135, 2005

[14] J.C. Miñano, J.C. González, "New method of design of nonimaging concentrators", Appl. Opt. 31, pp. 30513060, (1992)

[15] Juan C. Miñano, Pablo Benítez, Wang Lin, Fernando Muñoz, José Infante, Asunción Santamaría, "Overview of the SMS design method applied to imaging optics", Proc. SPIE 7429, 74290C (2009).

[16]P. Benítez and J. C. Miñano, "Ultra high-numerical-aperture imaging concentrator," J. Opt. Soc. Am. A 14, 1988-1997 (1997) http://www.opticsinfobase.org/abstract.cfm?URI=josaa-14-8-1988

[17] Juan C. Miñano, Juan C. González, and Pablo Benítez, "A high-gain, compact, nonimaging concentrator: RXI," Appl. Opt. 34, 7850-7856 (1995)

[18] Juan C. Miñano, Pablo Benítez, Wang Lin, José Infante, Fernando Muñoz, and Asunción Santamaría, "An application of the SMS method for imaging designs," Opt. Express 17, 24036-24044 (2009) http://www.opticsinfobase.org/oe/abstract.cfm?URI=oe-17-26-24036

[19] R.K. Luneburg, Mathematical Theory of Optics, (Univ. Calif.Press, Berkeley 1964).

[20] O.N. Stravoudis, The Optics of Rays, Wavefronts and Caustics, (Academic Press, New York, 1972).

[21] R.K. Luneburg, Mathematical Theory of Optics, (U. California, Berkeley, 1964)

[22]T. Levi-Civita: Atti della Reale Accademia dei Lincei. Rendiconti della Clase de ScienzeFisiche, Matematiche e Naturali 9, (1900) 185 and 237.

[23] K. Schwarzschild, Astronomische Mitteilungen der Königlichen Sternwarte zu Göttingen 10, 3 (1905), Reprinted: Selected Papers on Astronomical Optics, SPIE Milestone Ser. 73, 3 (1993).

[24] G.D. Wassermann and E. Wolf, "On the Theory of Aplanatic Aspheric Systems," Proc. Phys. Soc. B 62, 2-8 (1949).

[25] W.T. Welford, “Aplanatism and Isoplanatism," Progress in Optics 13, 267-293, (1976).

[26]L. Mertz, "Geometrical design for aspheric reflecting surfaces," Appl. Opt. 18, 4182-4186 (1979).

[27]L. Mertz, "Aspheric potpourri," Appl. Opt. 20, 1127-1131 (1981).

[28] D. Lynden-Bell, "Exact Optics: A Unification of Optical Telescope Design," MNRAS 334, 787-796 (2002)

[29] R.V. Willstrop, D. Lynden-Bell, "Exact Optics - II. Exploration of Designs On- and Off-Axis," MNRAS 342, 33-49(2003) 